\title{
Compuflo Epidural Instrument is capable of discriminating false LOR during difficult epidural placement for labor analgesia
}

\section{Camorcia ${ }^{1}$, A. Coccoluto ${ }^{1}$, M. Velardo ${ }^{1}$, U. Bitossi ${ }^{2}$, P. Livi ${ }^{2}$, A. Di Filippo ${ }^{2}$ \\ ${ }^{1}$ Città di Roma - Roma (Italy), ${ }^{2}$ Careggi Hospital - Firenze (Italy)}

\begin{abstract}
Background and Goal of Study: The CompuFlo® Epidural Instrument is a computer-controlled pump that can measure the pressure of human tissues in real-time at the tip of a needle. This so called exit pressure regulates the flow rate dispensed by the pump.

This instrument provides an audible and a visual display of exit-pressure helping the anesthesiologist to identify different lumbar tissues, the ligamentum flavum and the epidural space.

It has been already successfully used for routine cases 1 . The aim of our study was to evaluate the performance of Compuflo when used by trainees as a rescue tool in difficult epidural placement.
\end{abstract}

Materials and Methods: We obtained IRB approval and patient informed consent. For the purpose of this study we enrolled only parturients for whom two needle re-insertions were required by trainees during the epidural procedure for labor analgesia. Compuflo was used from the third attempt as a rescue tool. The primary goal was to gauge the ability of the Compuflo to differentiate false LOR. The number of epidural attempts and successful epidural analgesia (VAPS $<10$ after 20 minutes) were also noted. A sample size of 50 observations to set $80 \%$ test power and a $95 \%$ significance was required. Statistical analysis was performed with unpaired t-test.

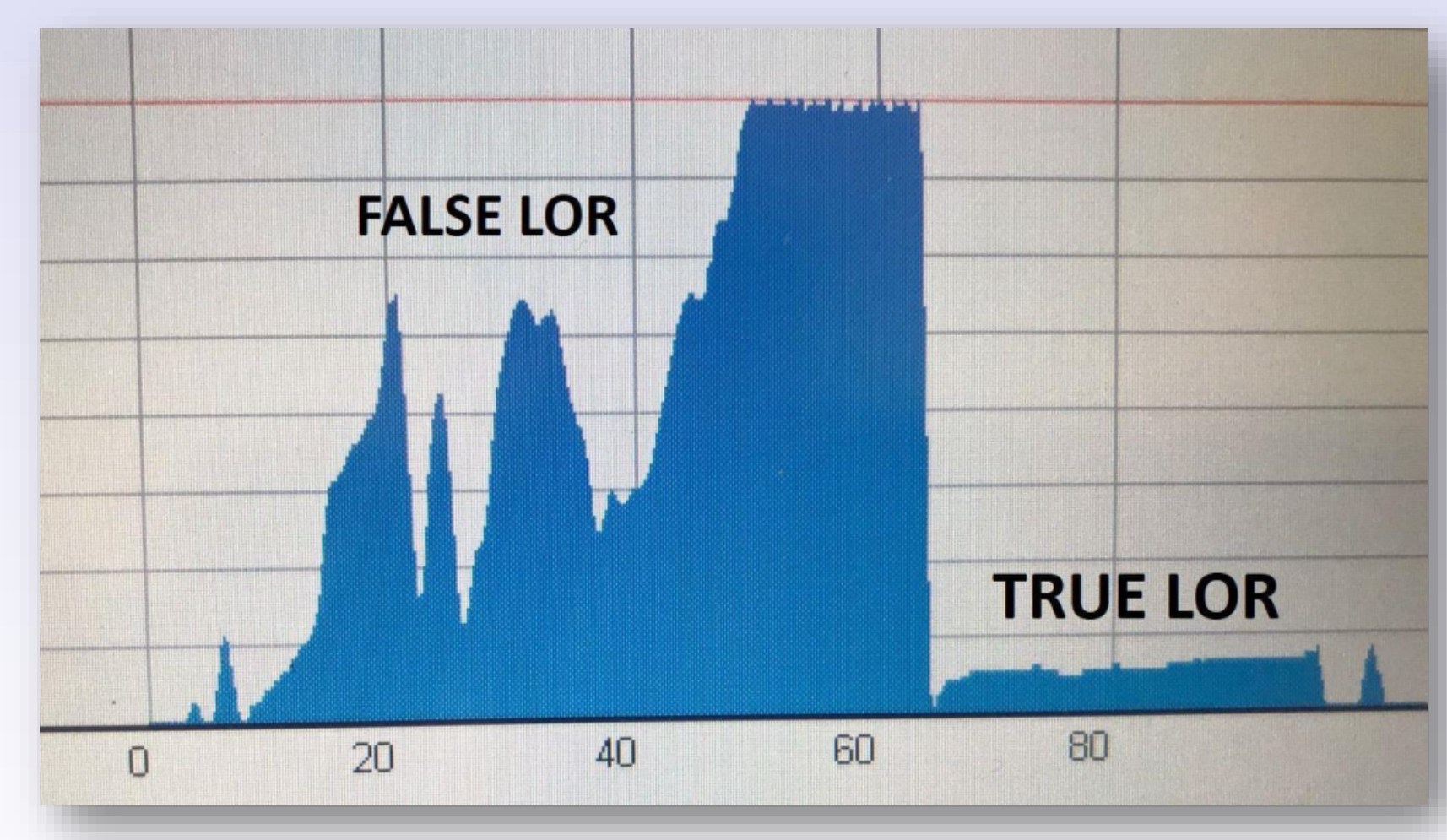

True-LOR: a sudden and sustained (> 5 seconds) drop in pressure (typically greater than $50 \%$ of the maximum pressure), resulting in the formation of a "low and stable pressure plateau"

False-LOR: a drop in pressure (typically lasting 2-3 sec) followed by a rapid increase

Results and Discussion: We studied 56 consecutive patients. All the blocks performed with the Compuflo were successful after only one attempt in all the cases. The pressure curves of false LOR were significantly different from the true LOR (figure)

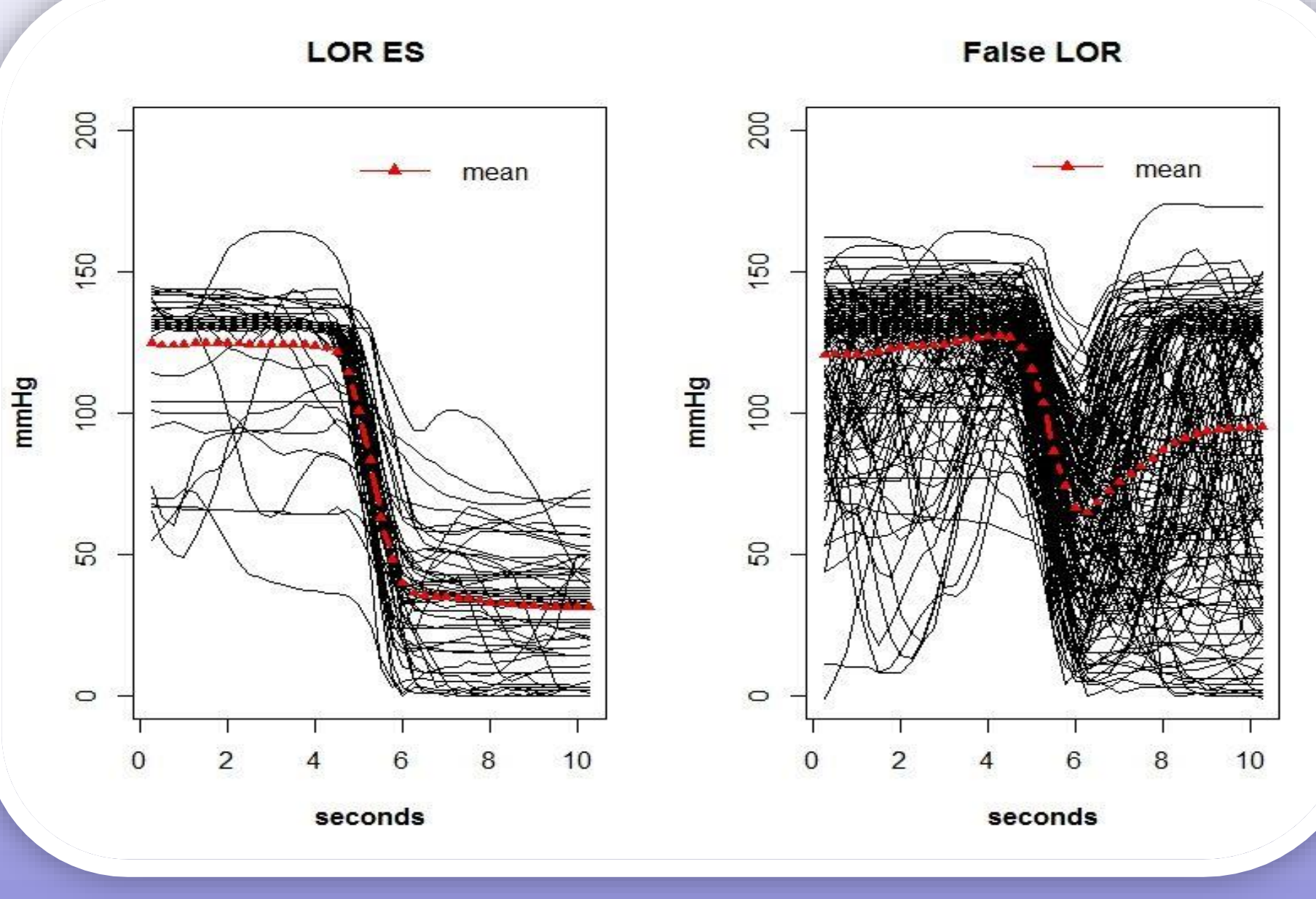

Conclusion: Compuflo can discriminate false from true LOR to identify the epidural space and therefore may assist the physician in difficult cases, reducing the number of attempts and producing successful epidural block. 